\title{
God, Time, and the Implicate Order Theory
}

\author{
SAMPSA KORPELA \\ University of Helsinki \\ sampsa.korpela@gmail.com
}

\begin{abstract}
In this article, the God's relationship to time is viewed from the perspective of modern physics. The purpose is to examine new perspectives by introducing a theory of time that has been unexplored in contemporary theology. The paper begins with an analysis of the two competing views of God's relationship to time: timelessness and temporality. They are reviewed from the perspective of the special theory of relativity. In contemporary theology, God's timelessness is usually combined with the block universe theory, which is based on the concept of unchanging spacetime. God's temporality is usually associated with presentism, which denies the concept of spacetime. This division reflects a central conflict in physics: the mainstream interpretation of the special theory of relativity treats time as unchanging spacetime, while quantum physics treats time as dynamic and flowing. To resolve this conflict between the ontologies of the special theory of relativity and quantum physics, the implicate order theory is introduced. The implicate order theory was developed by David Bohm (1917-1992), one of the most visionary physicists of the $20^{\text {th }}$ century. After introducing the theory, it is applied to the context of God's relationship to time. This produces interesting new opportunities for theological research.
\end{abstract}

Keywords: God and time, Theory of relativity, Quantum physics, Implicate order, Potentiality

\section{God and Time in Contemporary Research}

In this section of the paper, I first take a look at God's timelessness and then at God's temporality. I present a crucial counterargument to classical theism and, based on Einstein's special theory of relativity, analyze an answer to the counterargument. Then, I take a look at God's temporality from the perspective of the special theory of relativity. The goal of this first section is to outline the most crucial problems of the accounts of God's timelessness and temporality as they are usually presented in contemporary research, which I then seek to resolve in the second section by presenting the implicate order theory. 


\subsection{God's Timelessness in Classical Theism}

God's timelessness is closely tied to classical theism. From Antiquity to early modern times, classical theism was the dominant model of describing God's attributes. Lists of God's attributes usually contain the following: omnipotence, necessity, omniscience, perfect goodness, immutability, timelessness, simplicity, and impassibility.

What separates classical theism from temporal accounts of God (for example, personal theism) are the last four: immutability, timelessness, simplicity, and impassibility. These attributes form a special kind of combination in which they are strongly connected to each other. The concept of God's timelessness is strongly tied to His immutability: any succession in God's existence would lead to the conclusion that he is mutable. God's immutability (and thus timelessness) can also be derived from the concept of God's metaphysical simplicity: as God's simplicity is derived from Him being fully actualized potentiality, there can be no change in Him. There can be no unactualized potentiality in God, otherwise there would be potentiality for change and thus potentiality for succession (Mullins 2016, 47-63).

The whole existence of a timeless God is present to Him all at once. God's existence has no beginning, end, succession, temporal location, or temporal dimension. God's whole life exists for Him all at once. The concept of a timeless present can be applied to describe God's timelessness. The need to apply temporal terms to timeless concepts poses a challenge for the definition of timelessness (Mullins 2016, 47-49).

Although God's timelessness seems like a mystery, God's relationship to the time of our physical universe can be described by a classical analogy: the relationship between the center and the circumference of a circle. The center represents God's timeless now, whereas the circumference of the circle represents physical time. All moments of physical time exist equally from God's perspective (DeFlorio and Frigerio 2019, 211-212).

The most popular theory of time among the developers of classical theism is presentism. ${ }^{1}$ Although they are supporters of presentism, they also adopt the analogy of a circle and its circumference to describe God's relationship to physical time, which means that they think that all moments of the temporal universe exist simultaneously for God. For medieval classical theism, a serious

\footnotetext{
${ }^{1}$ According to presentism, only the present moment exists. The past no longer exists, and the future does not yet exist. Augustine (354-430), Boethius (480-524/525), and Thomas Aquinas (1225-1274) are three of the four most important developers of classical theism. In contemporary research, Augustine and Boethius are usually identified as advocates of presentism. Although many contemporary theologians and philosophers also consider Aquinas to be a presentist, there are also those who view him as an eternalist; see for example Costa 2019.
} 
problem lies in the attempt to connect presentism and God's timelessness. There is a logical contradiction in the claim that all moments of time are equally real from God's timeless perspective, but only the present moment is real from the temporal perspective. The contradiction is due to the fact that any moment of time cannot exist from one perspective if it does not exist from the other. This critique was actually not invented by modern theologians. The Franciscan theologian Duns Scotus (1266-1308) presented it in the late medieval times (Padgett 1992, 52-53).

This logical contradiction has been resolved in contemporary theology by applying a theory of time according to which all moments of time of the temporal physical universe are equally real; both from God's timeless perspective and from the temporal perspective. Eternalism, according to which all moments of the temporal universe exist eternally, is usually presented to enable this solution. A specific form of eternalism is the block universe theory. According to the block universe theory, four-dimensional spacetime, in which time is the fourth dimension in addition to the three spatial dimensions, is completely changeless (Mullins 2016, 147).

Next, I will examine the block universe theory to determine whether it can solve the aforementioned contradiction of classical theism. Advocates of the block universe theory often claim that it has an actual basis in physics, in the special theory of relativity. Thus, to examine the block universe theory, I take a closer look at its justifications by introducing some basic concepts of the special theory of relativity.

\subsection{Basic Concepts of the Special Theory of Relativity}

Einstein developed the special theory of relativity in 1905. According to the theory, objects do not travel merely through space but also through time. The speed of light (approximately 300000 kilometers per second in a vacuum) is the maximum speed that anything can travel. The speed of light must always remain the same for all observers (or, more generally, in all reference frames). Whenever an observer measures the speed of light, the result of the measurement must always be the same regardless of the speed at which the observer moves. Every observer has its subjective reference frame from which it perceives space and time. Two observers moving relative to each other and viewing the same event observe different spatial and temporal coordinates (also known as spacetime coordinates) for the event. Only the measurements of spacetime intervals between two events remain the same from the perspective of all reference frames. ${ }^{2}$ Measuring spacetime intervals requires combining the movement of

\footnotetext{
2 An event is defined as a point that has coordinates in three spatial dimensions and one temporal dimension. Thus, two events represent two different points in space and time (also
} 
objects through space and through time. This combined movement through space and time is what all observers can agree on. Retaining invariant spacetime intervals is usually referred to simply as retaining the invariant speed of light in all reference frames (Maudlin 1994, 34-57).

The observer-dependence of temporal and spatial measurements appears for example in the form of time dilation. ${ }^{3}$ The movement of objects occurs through both space and time; the faster the object moves through space, the smaller proportion of its movement occurs through time. Thus, time passes slower in the reference frame of a fast-moving object. Time dilation is hardly observable in everyday life because the slowing down of time is noticeable only when the speed of the object approaches the speed of light. However, with accurate enough measurement devices, time dilation can be observed even for objects whose speed is nowhere close to the speed of light. If an object reaches the speed of light, it does not experience the passage of time at all. Reaching the speed of light is possible only for objects that have no mass, such as photons (Greene 1999, 5658).

Since the speed of light is the maximum speed that any particle can travel, it is also the speed limit for causal effects. Thus, the speed of light limits the area of space that an observer can be causally connected within a certain timespan. This area is described by past and future light cones. If we choose the timespan $t$ to be for example one year, then the past light cone contains all events from which light can reach the observer - and thus causally affect the observer - in one year. Similarly, a future light cone can be constructed for the observer, containing all events that the observer can possibly causally affect in the chosen duration $t$. The observer is time-like connected with the area inside the light cones. The area outside the light cones is the absolute elsewhere. Events outside the light cone of the observer are causally disconnected from the observer since a light signal cannot travel fast enough to connect them. Causal disconnectedness can be defined by treating the reference frame of the observer as an event that takes place as a single point of spacetime. If the timespan between two events (the spacetime points of the observer and of the observed event) is less than the

called spacetime points or point events). A spacetime interval, the difference between two events, is $\mathrm{s}^{2}=\left(\mathrm{x}_{2}-\mathrm{x}_{1}\right)^{2}+\left(\mathrm{y}_{2}-\mathrm{y}_{1}\right)^{2}+\left(\mathrm{z}_{2}-\mathrm{z}_{1}\right)^{2}-\left(\mathrm{t}_{2}-\mathrm{t}_{1}\right)^{2}$ (derived from the Pythagorean theorem). A spacetime interval is more precisely expressed in the form $\mathrm{ds}^{2}=\mathrm{dx}^{2}+\mathrm{dy}^{2}+\mathrm{dz}^{2}-\mathrm{dt}^{2}$. When the spacetime coordinates of an event are transferred from one reference frame to another, the spacetime interval $\mathrm{s}^{2}$ remains invariant between the reference frames, and thus the spacetime interval is independent on the reference frame (Koperski 2015, 110).

3 Other observer-dependent physical measurements are the length and the mass of an observed object. The observer-dependence of the length of an object is called length contraction. The faster an object moves relative to the observer, the shorter it appears. The observerdependence of the mass of an object is called mass variation. The faster on object moves relative to the observer, the larger its mass is (Koperski 2015, 120). 
distance between the events divided by the speed of light, they are causally disconnected (Greene 2004, 70-72).

To define simultaneity between the observer and a causally disconnected event, a simultaneity relation between the reference frame of the observer and the area of spacetime belonging to the absolute elsewhere must be postulated. This simultaneity relation is described by the hypersurface of the present, also known as the simultaneity hyperplane. Different observers that move relative to each other disagree on the temporal order of the occurrence of events outside their light cones because the angle with which the hypersurface of the present cuts the spacetime depends on the relative speed of the observer. The greater the speed of the observer, the greater the angle in which it cuts spacetime. The difference between hypersurfaces of the present is usually negligible with relatively short distances, but with a great distance, even a small difference in relative speed causes the hypersurfaces between different observers to differ greatly (Greene 2004, 73). Picture 1 below depicts the effect of the speed of an observer on the hypersurface of the present.

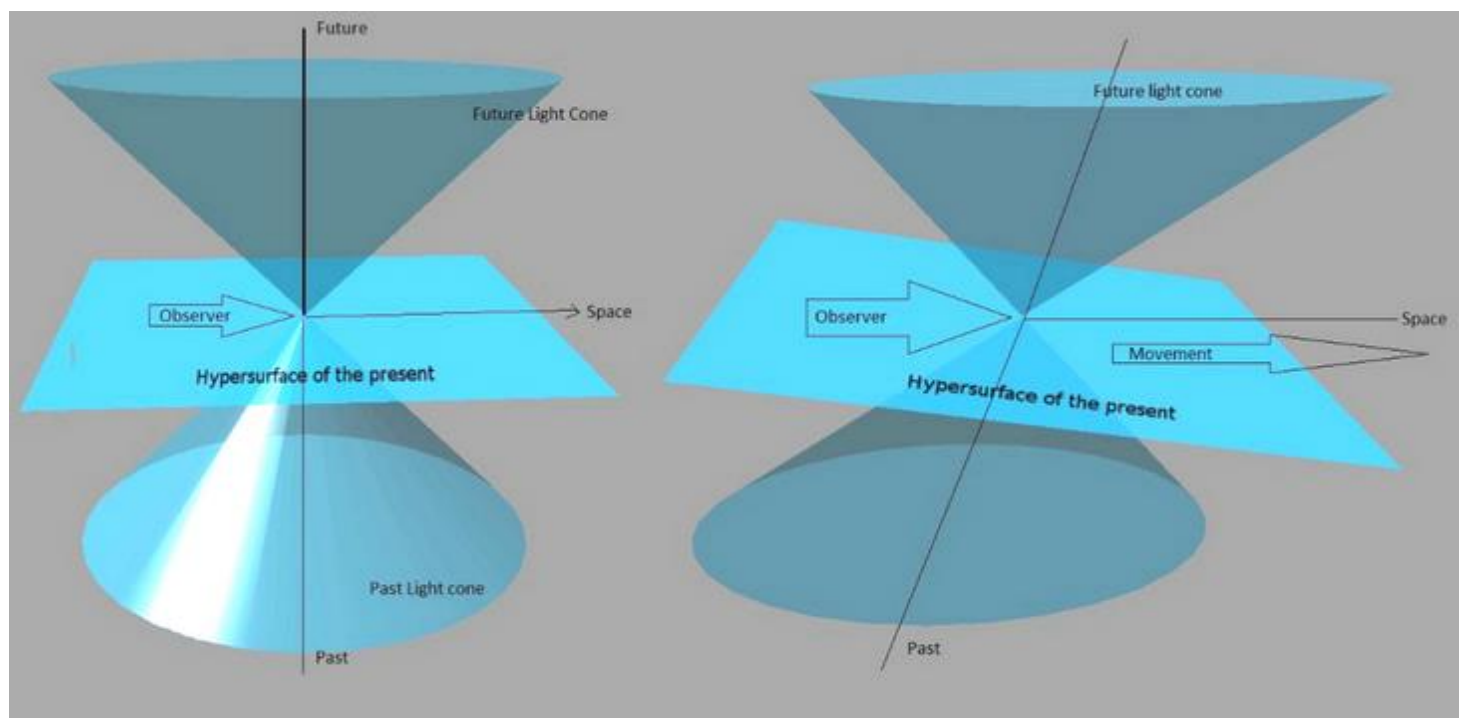

Picture 1: The light cone, the hypersurface of the present, and relative movement. For a moving observer, the light cone and the hypersurface is tilted. ${ }^{4}$

Soon after Einstein had published his special theory of relativity, Einstein's teacher, Hermann Minkowski (1864-1909), constructed his spacetime interpretation of the theory. Einstein immediately accepted the Minkowskian interpretation of the special theory of relativity. In contemporary physics, it has ever since remained the mainstream ontological interpretation of the theory. Minkowski reasoned that, because the simultaneity hyperplane of a moving

\footnotetext{
4 Encyclopaedia Galactica. "Light Cone". Accessed April 21, 2021. http://www.orionsarm.com/eg-article/49b476d5f018d.
} 
observer cuts spacetime at an angle and because there are observers moving at different speeds, there must be as many different hypersurfaces of the present as there are observers. From the perspective of an observer who is moving towards another observer who is stationary in relation to the moving observer, some events that are not yet real for the stationary observer may belong to the hypersurface of the present or even to the past of the moving observer. Thus, what is past or present for one observer may be the future for another observer. It would be logically contradictory for the events that are real from the perspective of the moving observer to be unreal from the perspective of the stationary observer. An event cannot be real in one reference frame and unreal in another (Dainton 2010, 329).

To avoid this contradiction, the same events must be real for both observers. Thus, the future of the stationary observer is already real even though those future events have not yet occurred from his perspective. It follows that past, present, and future moments of time must exist equally. Thus, the special theory of relativity implies the existence of the four-dimensional spacetime in which three spatial dimensions are merged with a time dimension. The fourdimensional spacetime is also called Minkowski spacetime, which in the philosophy of time is often called the block universe theory (Dainton 2010, 330).

\subsection{Is the Future of the Block Universe Pre-Determined?}

In contemporary theology, advocates of classical theism often point out that the Minkowskian interpretation of the special theory of relativity resolves the aforementioned dilemma that emerged from the combination of presentism and classical theism: that all moments of time exist from God's timeless perspective but that only the present moment exists from the temporal perspective. As the block universe theory implies that all moments of time from the beginning to the end of the universe exist equally, there is no problem with the assumption that all moments of time exist for God.

However, the solution based on the block universe theory comes with a serious philosophical and theological cost. As there is only one possible future, the existence of alternative futures is impossible. Thus, free will seems impossible. Because free will is a basic requirement for the existence of moral responsibility, it must somehow be included in order to make the block universe model theologically feasible (Koperski 2015, 115-116). Proponents of God's timelessness can defend the block universe theory by postulating that the one existing future follows causally from the temporal events of the universe, which contain acts of free will (Padgett 1992, 77).

Thus far, however, advocates of the block universe have been unable to construct a theory of time that would enable the existence of alternative possible 
futures in the block universe. ${ }^{5}$ Such attempts seem futile since the topological structure of the block universe, by definition, contains only one possible future. If there cannot be any alternative possible futures, no acts of free will are possible. Thus, the block universe theory leads to the pre-determination of the future (Koperski 2015, 115).

For the proponents of the block universe theory, a critical question remains unanswered: how can the existence of alternative possible futures be integrated into the concept of spacetime? It seems clear that, in order to enable the existence of alternative possible futures, the block universe theory has to be replaced with some other theory of time that allows the openness of the future. The paradigmatic solution in contemporary research on God's relationship to time abandons both the block universe theory and classical theism and combines presentism with God's temporality. Next, I will examine this solution, especially from the perspective of the special theory of relativity.

\subsection{Abandoning God's Timelessness}

In the early modern age, classical theism had to surrender its paradigmatic position to God's temporality. Proponents of God's temporality abandon the four key attributes of classical theism: immutability, simplicity, timelessness, and impassibility. Discarding timelessness means that God is temporal. A temporal God exists in the present moment and experiences the flow of time at the same rate as created beings. God is eternal (also called sempiternal), meaning that His life is without a beginning and an end (DeWeese 2002, 56).

As the temporal universe is contingent but God's existence necessary, God is not usually thought to exist essentially in the physical time of the universe (Leftow 1991, 34). Instead of existing essentially in physical time, God is thought to exist essentially in metaphysical time, which is a more fundamental order of time than the physical time of our universe. ${ }^{6}$ Metaphysical time flows at the same rate as physical time (Zimmerman 2001, 78).

5 There are contemporary advocates of classical theism who assume that eternalism and human free will can be reconciled (see for example Helm 2010 and Rogers 2017). However, they do not explicate a topological structure of time that would enable the reconciliation of eternalism and human free will. It is clear that the block universe theory is not suitable for the purpose. In the last subsection of the paper, I formulate an eternalist account of branching time that incorporates human free will.

6 The doctrine of Christianity assumes that our universe is created by God, and thus it has a beginning. Also, in physics, the paradigmatic explanation for our universe is the Big Bang theory, according to which our universe has a beginning. Thus, both Christian doctrine and natural sciences support the idea that our physical time has a beginning. As God is a necessary and everlasting being, He cannot exist essentially in physical time. Thus, God must exist essentially in metaphysical time (DeWeese 2002, 56). 
In contemporary theology, presentism is usually seen as the only theory of time that is compatible with God's temporality. ${ }^{7}$ Presentism states that only the present moment is real and that past and future moments do not have real existence. Also, time has a real flow; the flow of time is not subjective or illusionary. The Minkowskian interpretation, which is the mainstream interpretation of the special theory of relativity, contradicts presentism by claiming that all moments of time exist equally, and the flow of time and the existence of the present moment are only subjective phenomena.

The most popular way to explain presentism in the context of the special theory of relativity is to abandon the Minkowskian interpretation of the special theory of relativity and replace it with the neo-Lorentzian interpretation. According to the neo-Lorentzian interpretation of the special theory of relativity, time dilation can be explained by the slowing down of physical processes that follow from the increase of the length of the route that electromagnetic signals need to travel when an object moves faster. The neo-Lorentzian interpretation denies that the increase of the speed of an observer actually causes time to slow down for the observer. Time is not merged with space, and thus the concept of spacetime is also denied. The denial of spacetime also eliminates any reason to think that the flow of time is not real (Craig 2001, 184).

Since the neo-Lorentzian interpretation of the special relativity is empirically indistinguishable from the Minkowskian interpretation, it is certainly allowed by physics. Thus, the neo-Lorentzian interpretation succeeds in defending presentism. However, the neo-Lorentzian interpretation comes with a cost as well. To defend presentism, one must make the assumption that contemporary physics is mistaken when assuming the reality of relativistic concepts, such as time dilation and the merging of space and time.

For a closer integration of theology and physics, other options besides presentism should be examined. God's temporality is not necessarily tied to presentism even though the two have been closely associated to one another in contemporary research of God's relationship to time. In the next section of this article, I show that there is an alternative option available for the advocates of God's temporality: the implicate order theory. With some modifications, the implicate order theory can also be applied by the advocates of God's timelessness.

7 There are also other accounts with real flow of time that can be combined with God's temporality, such as the growing block theory. The growing block theory postulates that the past and present are real but the future is not yet real. However, as the contemporary research of God's relationship to time is mostly focused on the juxtaposition of presentism and block universe, I treat presentism as the default account of God's temporality. In this paper, I also show that the implicate order theory can be applied to reformulate God's temporality. 


\section{Quantum Physics and the Concept of Potentiality}

In this section, I examine a theory of physics that can combine the concepts of spacetime and the real flow of time: Bohm's implicate order theory. The theory has been extensively discussed in the philosophy of physics, but the discussion has not yet been extended to the research of God's relationship to time. After introducing the implicate order theory, I explore its theological possibilities. I attempt to apply the theory to resolve the problems outlined in the first section: the lack of alternative possible futures in the theory of God's timelessness and the lack of spacetime in the theory of God's temporality.

\subsection{Unifying the Real Flow of Time with Spacetime}

In the first section, the examination God's relationship to time from the perspective of the special theory of relativity seemed to lead to the conclusion that the ontologies of presentism and the block universe theory contradict each other. The examination identified the central dilemma to be: how can the flow of time and the existence of alternative futures be integrated with the concept of spacetime?

A key observation is that the contradiction between presentism and the block universe closely resembles the contradiction between the ontologies of two central theories of contemporary physics: the special theory of relativity and quantum physics. The ontology of the block universe theory is based on the Minkowskian interpretation of the special theory of relativity. All moments of time are equally real, and the flow of time is only an illusion. The ontology of quantum physics contradicts the Minkowskian interpretation of the special theory of relativity. Quantum physics implies that there is a real flow of time and a real division to the past, present, and future. Instead of things existing eternally in a static four-dimensional spacetime, they become real at the present moment (Stapp 1985, 264).

To unify the ontologies of the special theory of relativity and quantum physics, one would have to abandon both the ontologies of presentism and the block universe theory and replace them with an alternative theory of time. In this unifying theory, relativistic concepts such as time dilation and spacetime are not abandoned like they are in the neo-Lorentzian interpretation. Also, the real flow of time and the existence of alternative possible futures are not abandoned like they are in the Minkowskian interpretation. Instead, the concept of spacetime is integrated with the concepts of the flow of time and a real present moment. 


\subsection{The Implicate Order Theory}

There is a theoretical framework in physics that is designed to reconcile the ontologies of the special theory of relativity and quantum physics: Bohm's implicate order theory. The theory is based on the idea that the successful unification of the theories requires abandoning the mechanistic framework of the special theory of relativity. ${ }^{8}$ A holistic framework is required to successfully unite the special theory of relativity and quantum physics at the ontological level. According to the holistic framework, each part of the universe is connected to the wholeness of the universe on a deeper level (Bohm 1980, 138-149; 176).

Bohm calls this deeper level the implicate order (derived from the word "implicit"), and from the implicate order arises our three-dimensional observed reality, which he calls the explicate order (derived from the word "explicit"). The implicate order contains all past and future moments, while the explicate order contains the present moment. Bohm also postulates that behind the implicate order, there is a multi-dimensional super-implicate order, which contains all possible timelines of the universe in a potential state, having a real but unactualized existence. ${ }^{9}$ Only one of the potential timelines becomes actualized through the flow of time. The actualized present moment is the explicate order, the three-dimensional present moment of the universe (Bohm and Hiley 1984, 258).

The implicate order theory resolves the aforementioned problem associated with the block universe: the pre-determination of the future, which follows from the existence of only one possible future. With the implicate order theory, the topology of the future of spacetime has the shape of a branching tree of potential

\footnotetext{
${ }^{8}$ According to Bohm's definition, the mechanistic framework has three key characteristics. First, the whole material universe is reducible to elementary particles (or fields that give existence to the particles). Second, the universe is the sum of these particles (or fields) and nothing more. These particles are separate to and independent of each other and they are not connected to the wholeness on a deeper level. Third, the interaction between the particles is purely mechanical, and thus the only relationship between the particles is through external, causal influence. This external causality corresponds only to classical causality, whereas quantum physics allows nonlocal causality (Bohm 1986(i), 15).

${ }^{9}$ Bohm successfully constructed a mathematical formulation of the super-implicate order for boson fields (for example, the electromagnetic field). The particle-like properties that the electromagnetic field has in the form of photons emerge as a result of the action of the superimplicate order, when energy is concentrated into a quantum packet from the area of the whole electromagnetic field. For the fermionic particles that constitute matter, Bohm did not succeed in creating a similar mathematical formulation (Pylkkänen 2007, 174-175). Other authors have attempted to complete the theory by expanding the mathematical formulations to fermionic particles. After Bohm's passing, there have been many advances in formulating the theory. However, there is no consensus among physicists whether these attempts can be considered successful. For a notable attempt, see Nikolic 2005.
} 
futures instead of only one available future. Since future potentialities are available in the present moment, the future is not pre-determined.

Another central difference compared to the block universe is that there is an objective present moment. The explicate order represents the present moment, which is objectively real. Thus, the flow of time is not only a subjective phenomenon (Bohm and Hiley 1984, 272). The justification for the existence of spacetime is no longer that all moments of time must exist equally, as the block universe theory entails. By combining the theory of special relativity with quantum physics, the implicate order theory gives the concept of spacetime a completely new ontological justification: the division between actual and potential.

\subsection{The Actualization of Potentialities from the Super-Implicate Order}

In his most advanced articulations of the implicate order theory, Bohm formulates the actualization of potentialities in the framework of the quantum field theory. ${ }^{10}$ The quantum field theory postulates that the existence of quantum fields is more fundamental than the existence of individual particles. Each type of particle (electrons, photons etc.) has its own corresponding quantum field that spreads through the whole universe (Bohm and Hiley 1993, 230-236).

The quantum field theory treats particles as a series of repeated energy spikes of the corresponding quantum field. A particle seems like a stable entity, but is actually constantly re-created by extremely rapid concentrations of energy from the quantum field. Without this constant re-creation that is guided by the implicate order and the super-implicate order, all physical objects would instantly cease to exist (Cobb 1985, 158). This constant re-creation of particles is the process of actualization.

\footnotetext{
${ }^{10}$ The quantum field theory is the most accurate quantum theory of contemporary physics (or, more accurately, one should speak of quantum field theories as there are many versions of the theory). The central motivator for the quantum field theory is that the Schrödinger wave function, which describes the dynamic evolution of a quantum system, is not Lorentz invariant. To maintain Lorentz invariance, the spacetime coordinates of an event must be transformable from one inertial reference frame (a reference frame moving at a constant speed) to another. This transformation of spacetime coordinates between inertial reference frames is called Lorentz transformation, and transformability of the coordinates is called Lorentz invariance. Due to the low speed of most particles when compared to the speed of light, the non-Lorentz-invariance of traditional quantum physics does not usually cause serious inaccuracies to predictions of the evolution of quantum systems. However, because nowadays quantum physics examines highspeed particles, especially particles accelerated in large hadron colliders, relativistic treatment is necessary for gaining accurate results. Also, the treatment of electromagnetic fields (in which light particles, photons, are manifest) is incompatible with non-relativistic quantum physics and thus requires quantum field theoretical treatment (Kuhlmann 2012).
} 
A more familiar example of actualization is the collapse of the wave function. According to the usual interpretation (the Copenhagen interpretation) of quantum physics, the collapse of the wave function represents the process of actualization. Werner Heisenberg (1901-1976), one of the early central developers of quantum physics, suggests that the existence of potentialities in a single-particle system is described by the Schrödinger wave function. Before the collapse of the wave function, a quantum system possesses all its quantum states simultaneously. This simultaneous existence of all states of the quantum system reflects the existence of potentialities. When the quantum system takes a definite value in the collapse of the wave function (which is caused by a measurement or by an observation of the system), a potentiality becomes actualized (Heisenberg 1958, 53).

Bohm agrees that the wave function contains the potentialities. However, there are three ways in which Bohm advances Heisenberg's view of the concept of potentiality. First, there is no such thing as the collapse of the wave function. Particles have real existence even when they are not measured or observed. Second, Bohm postulates that, instead of separable single-particle systems, there is only one, universal quantum system: the implicate order, which contains potentialities for the next present moment. Single-particle quantum systems are only approximations: any quantum subsystem of the implicate order is inseparable from the whole as the whole surrounding universe affects every subsystem. ${ }^{11}$ Third, all potentialities exist eternally in the super-implicate order, which directs the quantum fields through the implicate order. The superimplicate order contains all potential states of the universe from its beginning to its end, whereas the implicate order contains all potentialities for the next present moment. The implicate order mediates information from the super-implicate order to the quantum fields, directing the energy spikes of the quantum fields, which manifest as stable particle-like entities in the explicate order (Bohm 1986(ii), 154-155).

What is the ontological status of the potentialities? The super-implicate order contains all the potential ways in which the future can unfold. The potentialities are ontologically real, existing in the multi-dimensional structure of the superimplicate order. However, the potentialities have a clear ontological difference compared to actualized events. Actualized events consist of energy spikes of the

\footnotetext{
${ }^{11}$ In contemporary physics, a more familiar name for the implicate order is "the wave function of the universe". Because the form of the quantum field is affected by every particle in the universe, the wave functions of subsystems inspected in empirical quantum physics are approximations. The wave function of the universe is a complex system that consists of a vast amount of quantum subsystems, each of which is affected by its surroundings (Durr et al. 1992, 858-866).
} 
quantum fields, whereas potential events consist of all the possible states of the quantum fields. ${ }^{12}$

\subsection{The Implicate Order Theory Combines Growing Block and Branching Spacetime}

To complete the examination of the implicate order theory, there is one more aspect to consider: the part of the implicate order that contains the actualized past. Bohm explains that past events are in an enfolded state contrary to present events, which are unfolded in the explicate order. All past moments of time have a real but enfolded existence in the implicate order of the past (Bohm 1980, 205). Their ontological status differs from the events that are currently being actualized in the explicate order. This way, their status is different than in the block universe, which simply treats all moments of time as ontologically equal.

A central factor of the actualized past is its role in coordinating the actualization of the potentialities. The actualized past, which is enfolded back into the implicate order, determines which potentialities of the super-implicate order are available. Only those potentialities that are causally available from the perspective of the actualized past are available for actualization (Bohm 1985(i), 173). ${ }^{13}$ The whole process of actualization is summarized in picture 2 below.

\footnotetext{
12 The organization of potential states of the universe into a higher-dimensional structure is comparable to the functionality of a computer, which can store information and retrieve it from tables with coordinate numbers. Similarly, the information of the potential states of the physical universe could be stored into a multi-dimensional structure (Pylkkänen 2007, 171).

${ }_{13}$ Bohm's best-known theory, the pilot wave theory, postulates that quantum physics is deterministic. Any quantum-level indeterminism actually follows from hidden variables: unobservable variables that make quantum systems behave in seemingly random ways. Bohm's latest formulations of the implicate order theory unify the pilot wave theory with the quantum field theory. However, the implicate order theory is not completely deterministic. Bohm postulates that acts of human free will are not causally determined by the past states of the universe. In his attempt to explain how free will can be maintained in the framework of the implicate order, Bohm uses an analogy of the interaction between a player, a computer, and a display. According to the analogy, the explicate order is comparable to the events that occur on a display, the implicate order is comparable to the display, and the super-implicate order is comparable to the computer that arranges the things that are shown on the display. The "player", whose consciousness is tied to the interaction, sees the events of the explicate order occurring on the display and is immersed as a part of them. Without the role of consciousness, the implicate order and the super-implicate order would only execute a pre-determined program (Bohm and Peat 1987, 191-192).
} 
The super-implicate order

Contains all potential states of the universe from the end to the beginning of time in a timeless and unchanging branching structure

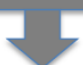

\section{The implicate order}

Contains all potential states of the next present moment, offering potentialities to the quantum fields

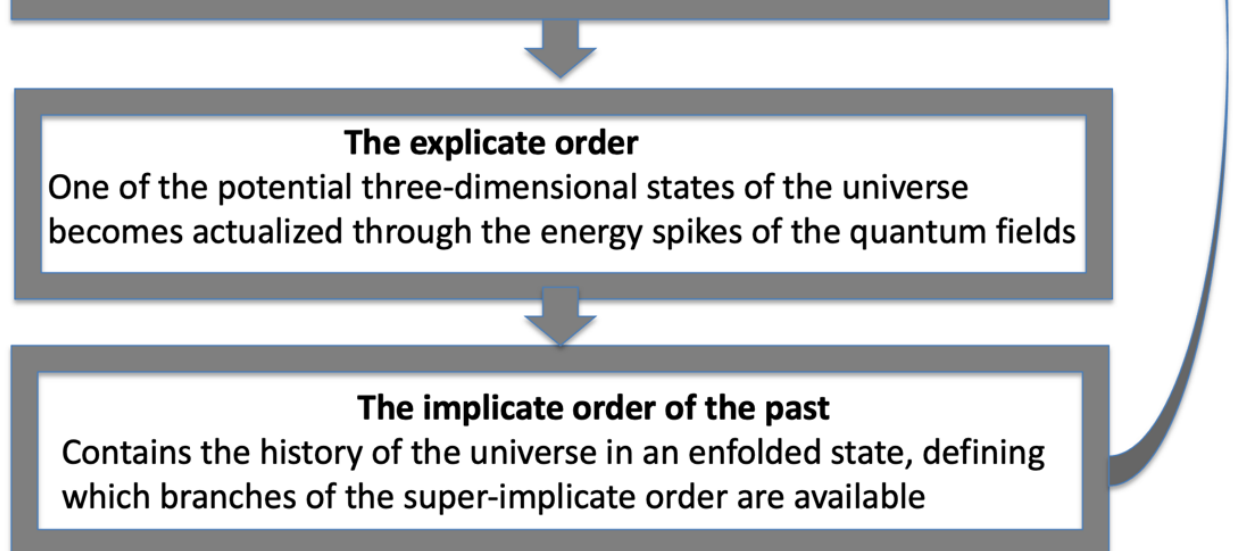

Picture 2: The process of the actualization of potentialities according to the implicate order theory.

Bohm mentions that the coordination between the past implicate order and the super-implicate order is crucial for maintaining the stability of particles. Without these higher-dimensional connections, energy spikes would appear randomly in the area of the quantum field, and the energy spikes would not generate patterns that form stable particles. This explanation gives the implicate order theory a significant advantage over other quantum field theories in ontological explaining power. Other quantum field theories cannot explain why the series energy spikes of the quantum fields are able to generate stable particles (Bohm 1985(i), 173).

Bohm never formulated the implicate order theory specifically in the form of a branching spacetime theory. He was more interested in presenting the physics of the theory than formulating it in the context of the philosophy of time. However, it is obvious that the version of the implicate order theory that contains the concept of the super-implicate order shares defining properties with branching spacetime theories. Although the super-implicate order is eternally unchanging from the eternal perspective, the branches that cannot causally follow from actualized history can be treated as "fallen off" when viewed from the temporal perspective. The past spacetime of the implicate order is similar to the growing block universe. In this way, the implicate order theory is a hybrid of 
two theories of time: growing block universe (past) and branching spacetime (future). The structure of branching spacetime (and its comparison to presentism and the block universe) can be seen in picture 3 below. ${ }^{14}$

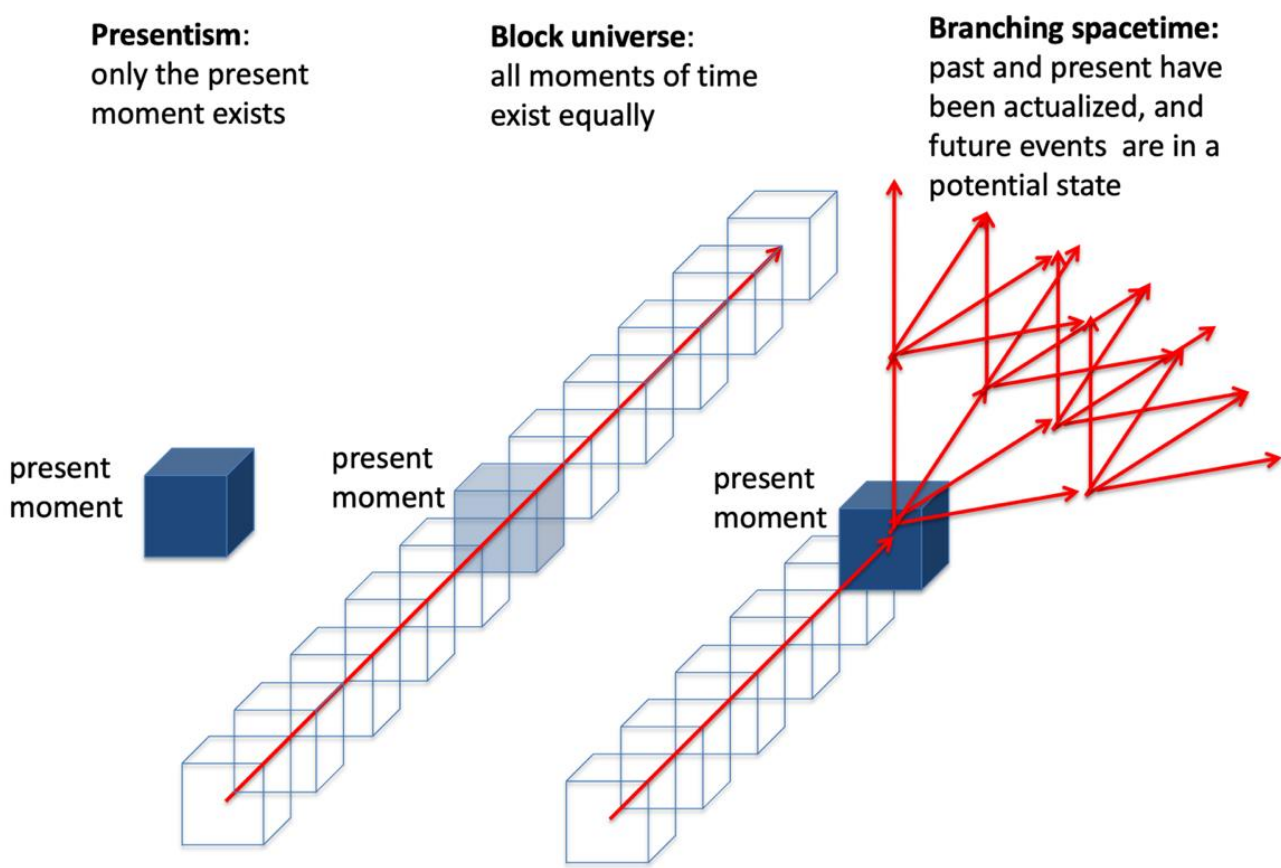

Picture 3: The structure of time according to presentism, block universe and branching spacetime theories. There is no objective present moment in the block universe.

In the next subsection, I examine the implications of the implicate order theory for God's relationship to time, and I present two alternative interpretations. The first interpretation postulates that the network of potentialities exists eternally and that there is an objective flow of time that God experiences at the same rate as created being do. The second interpretation postulates that, from God's eternal perspective, spacetime appears to Him as completely actualized. The flow of time is experienced by temporal observers who participate in the passage of time of the temporal universe from their own reference frames.

\subsection{The First Interpretation: God's Temporality}

In the first interpretation of the implicate order theory, I take Bohm's theoretical framework in its original form and apply it to God's relationship to time. From

\footnotetext{
${ }^{14}$ Branching spacetime theories have been discussed for example in Belnap 1992 and McCall 1994. For a discussion of branching spacetime in theological the context of theology, see for example DeFlorio and Frigerio 2019.
} 
the perspective of a temporal observer, the structure of time is a combination of growing block and branching spacetime. In the present moment, a future from among all the potential futures becomes actualized with the flow of physical time. As Bohm postulates that spacetime has an objective present moment, it is natural to assume that this objective present moment is God's perspective on physical time.

God created the potentialities at the moment of creation. From the eternal perspective, the super-implicate order remains unchanging. God's perspective on the unchanging super-implicate order represents His eternal perspective on time. The eternal perspective differs from the timeless perspective of the God of classical theism, which entails God's immutability. The postulation that potentialities exist eternally for God does not imply that He is immutable. Instead, since God participates in the flow of physical time, He cannot be immutable. An interpretation of the theory that enables God's immutability is presented in the next subsection.

This first interpretation might appeal to advocates of God's temporality. The structure of time shares similarities with presentism: there is an objective present moment and an objective division to the past, present, and future. However, adopting the implicate order theory instead of presentism offers several theological advantages:

1. It offers a theory of time that accepts the concept of spacetime, which is central in physics, and updates the concept to incorporate the quantum field theory, which is the most advanced quantum theory.

2. Treating particles as energy spikes of the quantum fields instead of permanent objects allows new opportunities for defining the concept of continuous creation. Without the constant actualization of potentialities from the super-implicate order, all physical objects would cease to exist instantly.

3. It helps explain how God knows future potentialities and thus can help define God's foreknowledge of the future. The knowledge of potentialities points towards Molinism as the account of God's foreknowledge.

4. The ability to see potentialities allows God to pre-arrange temporal events as He wills, especially if combined with the idea that God has the power to decide which potentialities become actualized whenever He wills to do so. This offers new opportunities for defining the concepts of creatio ex nihilo and divine providence.

Since Molinism has been questioned in contemporary theology, the validity of the last two advantages is debatable. Molinism postulates that before the moment 
of creation, God had a type of foreknowledge called middle knowledge, which included all the possible states of the universe and all choices of human free will in each of the possible states. Molinism also attempts to explain divine providence by postulating that, with the knowledge of future potentialities and the choices of human free will, God can actualize a universe that best suits His needs (DeFlorio and Frigerio 2019, 158-161).

However, there is a considerable drawback to Molinism. The principle of grounding states that God cannot foreknow the acts of human free will unless His knowledge is grounded in actual choices made by humans. The advocates of the principle of grounding claim that it is difficult to conceive what other grounding there could be than the actual choices of free will (DeFlorio and Frigerio 2019, 176-180). The implicate order theory lacks the grounding of God's knowledge of choices of human free will. The choices of free will are actualized in the present moment, and thus they do not exist before the actualization of potentialities. Thus, if the principle of grounding is adopted, the implicate order theory does not help explain how God can know the choices of human free will. God's knowledge of the super-implicate order only satisfies the requirement of His knowledge of all the possible states of the universe but does not imply that He knows the actual choices of free will.

In the framework of this first interpretation, the requirement of grounding of the knowledge of acts of human free will implies a diminishing of God's providential power. If God has no knowledge of actual choices of humans, He cannot have complete power to pre-organize future events as He wills. Such capacity would require taking into account choices of free will. However, it is debatable whether God needs to have such providential power and foreknowledge. One could as well assume open theism, according to which God has no definite knowledge and no perfect control of the future.

Alternatively, one can dispute the claim that God's knowledge of acts of human free will requires grounding in the real existence of future events. If the principle of grounding is rejected, this first interpretation of God's relationship to time in the framework of the implicate order theory seems satisfactory. However, in the case that the principle of grounding is adopted, an alternative explanation of God's foreknowledge and providential power is required.

To enable the principle of grounding, one might add the assumption that from God's eternal perspective, spacetime exists eternally as actualized from beginning to end. However, this would not enable Molinism, which entails that God knows all the choices of free will in all possible timelines of the universe. To explain middle knowledge, it is not enough that God knows which one of all the potential timelines will be actualized. God's knowledge of acts of human free will must be explained also in all the potential timelines that will never be actualized (DeFlorio and Frigerio 2019, 181). However, postulating that the whole 
actualized universe exists for God from beginning to end allows the timeless solution for explaining God's foreknowledge. This alternative is presented in the next subsection

\subsection{The Second Interpretation: The Two-Dimensional Theory of Time}

The second interpretation applies the implicate order theory to God's relationship to time by postulating that physical time has two dimensions: temporal and eternal. I choose to call this version of the theory the twodimensional theory of time. This interpretation is inspired by Bohm's theory, but it adds an additional layer to it: God's eternal perspective on spacetime that is completely actualized from the beginning of time to the end of time. Bohm had no reason to postulate the existence of a completely actualized spacetime as he did not take into account God's eternal perspective on time. This eternal perspective on time resembles God's timelessness in classical theism, and thus it might be embraced especially by advocates of God's timelessness. ${ }^{15}$ The division of time into temporal and eternal dimensions adds a key component that is missing in the block universe version of classical theism: the incorporation of free will into the concept of spacetime.

Theories of two-dimensional time have been presented before, although not in the form of a theory of branching time. Two well-known theories share similarities with my theory. The first is Anselm of Canterbury's (1033-1109) version of classical theism. ${ }^{16}$ The second is German theologian Karl Heim's (18741958) theory of two-dimensional time. ${ }^{17}$ With my version of the two-dimensional theory of time, I add to these theories what they lack: by using Bohm's theoretical

\footnotetext{
${ }^{15}$ I choose to call God's perspective of actualized spacetime eternal instead of timeless in order to underline the idea that the theory of two-dimensional time does not necessarily have to be combined with classical theism. It could as well be combined with God's temporality. One could discard God's immutability, at least in the form that it appears in classical theism, and still retain the idea that the whole actualized spacetime exists for God.

${ }^{16}$ Anselm is one of the four most important developers of classical theism. He thinks of God's timelessness as a higher dimension, in which our temporal universe is located. Thus, each temporal moment is actually present to God simultaneously, which explains God's foreknowledge of the future. Even though most contemporary researchers of God's relationship to time think that Anselm supports the block universe theory, Leftow notes that Anselm also assumes that the flow of time is real. It seems that, according to Anselm, the universe is static from God's timeless perspective but that the flow of time is real from the temporal perspective. However, Anselm does not explain how these two seemingly contradicting positions can both be true. He only refers to God's transcendence as an explanation (Leftow 1991, 183-199).

${ }_{17}$ The German theologian Karl Heim (1874-1958) was a close friend of Einstein. Based on the relativity theory, Heim postulated that the physical time of our universe has two dimensions: the temporal dimension of created beings and the eternal dimension of God, for whom the whole spacetime exists as an entity. For a summary of Karl Heim's thinking, see Eerikäinen 2000.
} 
framework, I explicate the relationship between temporal and eternal perspectives of time.

Branching time enables the timeless solution to resolve the contradiction of God's foreknowledge and human free will. The timeless solution is based on the idea that, as God's foreknowledge of the future is timeless, it does not temporally precede the acts of human free will. Thus, human free of will is not overruled by God's infallible knowledge of the future. Also, to maintain human free will, God's knowledge of the future must causally depend on the temporal process. This is called the principle of dependence. Due to the branching structure of time, human free will is maintained because future possibilities are available at each temporal moment (DeFlorio and Frigerio 2019, 240-250).

As spacetime has a fully actualized existence for God, the grounding principle of God's foreknowledge of the future is satisfied: God knows the future because all moments of time have an actualized existence for Him. However, for a temporal observer, there is a division into unactualized and actualized events and thus a division into the past, present, and future. The branching structure of spacetime allows the pre-existence of the future to be combined with human free will since the topological structure of spacetime is such that all possible futures are available from the perspective of a temporal reference frame. Thus, the twodimensional time interpretation of the implicate order theory seems to be able to explain how the topological structure of spacetime allows the combination of the pre-existence of the future with human free will.

As was stated in the first section of this paper, the presentist version of classical theism attempted to combine the real flow of time with God's timelessness. However, it suffered from the logical contradiction of future events being unreal from the temporal perspective and real from the timeless perspective. The theory of two-dimensional time resolves the contradiction with the division of events into the categories of actual and potential, which both belong to the category of existing events. In this way, an event can already be actualized from God's eternal perspective and still unactualized from the perspective of a temporal reference frame without causing a logical contradiction. From the temporal perspective, future events exist but are yet unactualized. The contradiction of the presentist version of classical theism is avoided.

From the perspective of the temporal observer, it seems that time flows dynamically, but time is actually tenseless (B-theoretical) in its objective form: any division into the past, present, and future is subjective. The temporal process does not generate new events. Instead, eternally existing events are introduced to a temporal observer. This process creates the subjective experience of the flow of time (Leininger 2020, 13-14). In the two-dimensional theory of time, the process of actualization is the introduction of eternally existing events to the observer. Thus, when inspected from the eternal perspective, spacetime seems 
like a block universe. However, the static spacetime is only half of the picture. As the principle of dependence states, future events that exist eternally for God are caused by the temporal process. The temporal process is real as well - but subjective, as it is tied to temporal reference frames.

An obvious objection to this theory is that the future cannot be both caused by the temporal process and pre-exist eternally "before" the actualization that occurs through the temporal process. The eternal and temporal perspectives seem to contradict each other, but the implicate order theory can coherently fit them together. From the perspective of the present moment of a temporal observer, all future branches of each next present moment are ontologically equally real in the implicate order. The implicate order is a universal wave function that contains all potential ways that the next present moment can be actualized. ${ }^{18}$ Through the process of actualization, only one of these potential branches becomes actual (see the picture 4 below).

18 The idea of the equal existence of each future branch is based on the quantum field theory. In his article concerning pre-space, Bohm interprets the implicate order as a vacuum state of empty space. Bohm's theory, like all versions of the quantum field theory, recognizes that the empty vacuum of space is not really empty but contains a huge amount of energy. All potential states of the next present moment of the universe exist simultaneously in the quantum fields. The simultaneous existence of all states is the zero-point energy of the quantum fields. The term zeropoint energy reflects the fact that physics can only observe energy states above the zero-point energy level of the quantum fields, in other words, energies that represent the actualized states of the fields. Thus, actualized particles are only a ripple on the surface of this vast sea of energy that is the vacuum state (Bohm 1985(ii), 196-197). The state of zero-point energy of the quantum fields is not only a mathematical formulation but can actually be experimentally verified by placing two plates extremely close together in a vacuum. Since only vacuum fluctuations with short wavelengths fit between the plates, the vacuum energy between the plates is diminished, and the pressure between the plates is less than the pressure outside the plates. The energy of the vacuum outside the plates exerts pressure on the plates, pushing them together. This pressure is called the Casimir effect (Lamoreux 1997). 


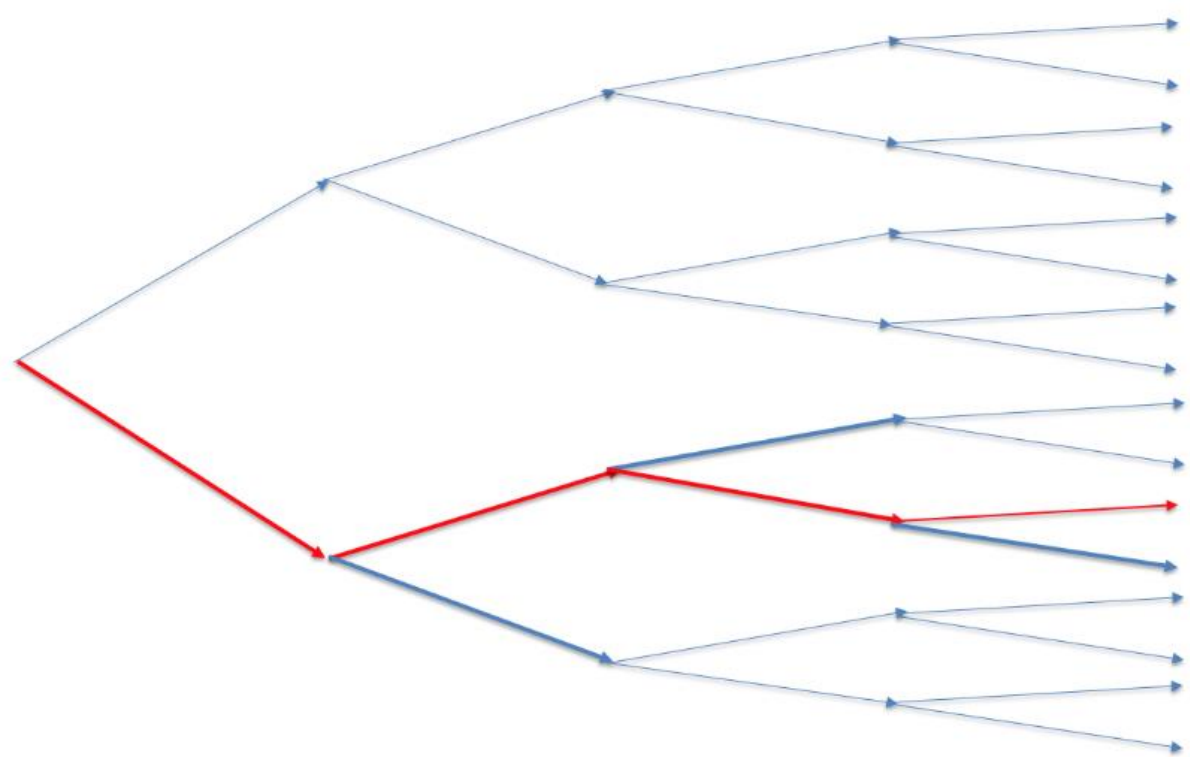

Picture 4: There are ontologically three types of branches: 1) Actualized branches (the red line), 2) Potential branches in the implicate order (the wider blue line), and 3) Potential branches in the super-implicate order (the narrow blue line). At each present moment, the potential branches depicted by the wider blue line are ontologically real.

From the perspective of the present moment of a temporal reference frame, the branch that is already chosen to be actualized from God's eternal perspective is ontologically indistinguishable from the branches that do not belong to the eternally existing actualized spacetime. However, as the future already exists for God, it is pre-determined. This makes human free will compatibilist instead of libertarian. According to compatibilism, the pre-determination of the future and the freedom of will are compatible. As the pre-determination of the future is caused by the temporal process of the actualization of potentialities, which includes the acts of human free will, compatibilism is sufficient to maintain real freedom of will and thus to allow real moral responsibility.

As a conclusion, it can be stated that the theory of two-dimensional time has all the same advantages as the first interpretation (see the list in the previous subsection that discusses the first interpretation of the theory). Additionally, the theory of two-dimensional time has at least two additional advantages that can be added to the list. First, the actualization of spacetime can help explain how God has foreknowledge of all future events: His knowledge is grounded in the actualization of the events. Second, God's knowledge of how events are actualized helps to explain His providential power. From God's eternal perspective, causal relations appear as relations that have already occurred. In the act of creatio ex nihilo, the perspective on actualized spacetime allows God to see how things unfold after the acts of human free will are taken into account. 
The network of causalities appears to God like a multi-dimensional puzzle. The ability to see all potentialities in the super-implicate order and the results of the temporal process of actualization give God the power to arrange events as $\mathrm{He}$ likes, especially if it is postulated that He has the top-down causal power to decide which potentialities become actualized whenever He wills to do so.

Also, the two-dimensional version of the theory makes the implicate order theory compatible with classical theism as spacetime is static from God's eternal perspective (or, according to classical theism, His timeless perspective). However, the theory is not limited to classical theism. One could as well postulate that God is temporal but that spacetime has a completely actualized existence for Him. God can live in His own metaphysical time, which does not need to be synchronized with the time of the physical universe. This way, the postulation that is common in contemporary theology, according to which God's metaphysical time must flow at the same rate as the time of the physical universe, is not absolutely necessary. Also, as God's foreknowledge of the future is based on the actualized future, the grounding principle becomes available to advocates of God's temporality.

\section{Conclusion}

In contemporary research, discussion about God's relationship to time has concentrated on a dichotomy between God's timelessness combined with the block universe theory and God's temporality combined with presentism. Neither of the accounts seems completely satisfactory. The account of God's timelessness suffers from the problem of incorporating free will into the block universe, and God's temporality combined with presentism suffers from the denial of spacetime.

It seems that to improve the overall coherence of God's relationship to time, and its coherence with contemporary physics, one must seek a solution outside the traditional pairs of timelessness / block universe and temporality / presentism. The juxtaposition between the block universe and presentism resembles the ontological contradiction between the special theory of relativity and quantum physics. To unite the perspectives, I have applied Bohm's implicate order theory since it is able to unite the ontologies of the special theory of relativity and of quantum physics. Applying the implicate order theory to God's relationship to time gives two alternative interpretations.

The first interpretation applies the implicate order theory directly, postulating that God experiences the flow of time at the same rate as humans. This makes the temporal account of God more justified from the perspective of physics by accepting the concept of spacetime instead of denying it. By applying the concept of actualization of potentialities, the implicate order theory modifies the concept 
of spacetime in a way that enables incorporating the real flow of time into it. The implicate order theory also adds the quantum field theory to the picture, giving the theory an even more rigid basis on physics.

The second interpretation adds a layer to the implicate order theory, postulating that God has an eternal perspective on time. This can resolve a major problem of God's timelessness: God's immutability has thus far been usually combined with the block universe, which has only one possible future, and thus it excludes the freedom of will. However, with two-dimensional time, spacetime is branching when viewed from the temporal perspective, and all possible alternative futures are in a potential state. Only from God's eternal perspective is spacetime fully actualized. This version of the theory might raise the objection that as the future already exists from God's perspective, it must be predetermined. This objection is answered by the notion that fully actualized spacetime follows causally from the temporal process of actualization.

The first interpretation is much simpler and also has a more rigid basis in physics as it follows the implicate order theory more closely. However, the implicate order theory does not take into account any theological perspective. Thus, it naturally excludes the possibility that God's perspective on time can be completely different from the temporal perspective. The assumption that, from God's eternal perspective, spacetime exists as completely actualized is not incompatible with the implicate order theory. It simply adds another layer to the theory. Added complexity is a price to pay for the theory of two-dimensional time.

Whether the theory of two-dimensional time has enough advantages over the simpler version that would justify its added complexity is a matter of further analysis. Regardless of which of the two alternative theories presented here is more justified, in the light of the argumentation presented in this paper, I find the implicate order theory to offer interesting opportunities for theology. Applying it to God's relationship to time can help define central theological concepts, such as the concepts of creation, divine providence, and God's foreknowledge of the future.

\section{Bibliography}

Belnap, Nuel. 1992. "Branching Space-Time." Synthese. 92:3. 385-434. https://doi.org/10.1007/BF00414289.

Bohm, David. 1980. Wholeness and the Implicate Order. New York: Routledge.

Bohm, David, Basil Hiley. 1984. "Measurement Understood Through the

Quantum Potential Approach." Foundation of Physics. 14:3. 255-274. https://doi.org/10.1007/BF00730211. 
Bohm, David. 1985(i). "Reply to Comments of John Cobb and David Griffin." In Physics and the Ultimate Significance of Time, edited by David R. Griffin. Princeton, New Jersey: University Press.

Bohm, David. 1985(ii). “Time, the Implicate Order, and Pre-Space.” In Physics and the Ultimate Significance of Time, edited by David R. Griffin. Princeton, New Jersey: University Press.

Bohm, David. 1986(i). "The Implicate Order: A New Approach to the Nature of Reality." In Beyond Mechanism: The Universe in Recent Physics and Catholic Thought, edited by David Schindler. Lanham: University Press of America.

Bohm, David. 1986(ii). "Hidden Variables and the Implicate Order." In Beyond Mechanism: The Universe in Recent Physics and Catholic Thought, edited by David Schindler. Lanham: University Press of America.

Bohm, David, David Peat. 1987. Science, Order, and Creativity: A Dramatic New Look Into the Roots of Science and Life. New York: Bantam Book.

Bohm, David, Basil Hiley. 1993. The Undivided Universe: An Ontological Interpretation of Quantum Theory. New York: Routledge.

Cobb, John. 1985. "Bohm and Time." In Physics and the Ultimate Significance of Time, by David R. Griffin. Princeton, New Jersey: University Press.

Costa, Damiano. 2019. "Aquinas, Geach, and Existence." European Journal for Philosophy of Religion. 11:3. 175-195. https://doi.org/10.24204/ejpr.v11i3.2837.

Craig, William L. 2001. God, Time and Eternity: The Coherence of Theism II: Eternity. Boston: Kluwer Academic Publishers. https://doi.org/10.1007/978-94-017-17151 .

Dainton, Barry. 2010. Time and Space. Montreal: McGill-Queen's University Press.

DeFlorio, Ciro, Aldo Frigerio. 2019. Divine Omniscience and Human Free Will: A Logical and Metaphysical Analysis. Cham, Switzerland: Palgrave Macmillan. https://doi.org/10.1007/978-3-030-31300-5.

DeWeese, Garrett. 2002. "Atemporal, Sempiternal, or Omnitemporal." In God and Time: Essays on the Divine Nature, edited by Gregory Ganssle, David Woodruff. https://doi.org/10.1093/acprof:oso/9780195129656.003.0003.

Durr, Deflef, Sheldon Goldstein, Nino Zanghi. 1992. “Quantum Equilibrium and the Origin of Absolute Uncertainty." Journal of Statistical Physics. 67:5/6. 843906.

Eerikäinen, Atso. 2000. Time and Polarity: The Dimensional Thinking of Karl Heim.

Dissertation. Helsinki: University Press.

Greene, Brian. 1999. The Elegant Universe: Superstrings, Hidden Dimensions, and the Quest for the Ultimate Theory. New York: Brockman Inc.

Greene, Brian. 2004. The Fabric of the Cosmos: Space, Time and the Texture of Reality. New York: Random House Inc.

Heisenberg, Werner. 1958. Physics and Philosophy. New York: Harper and Row. 
Helm, Paul. 2010. Eternal God: A Study of God Without Time. Oxford Scholarship Online. https://doi.org/10.1093/acprof:oso/9780199590391.001.0001.

Koperski, Jeffrey. 2015. The Physics of Theism: God, Physics, and the Philosophy of Science. Chichester, UK: John Wiley \& Sons.

https://doi.org/10.1002/9781118933800.

Kuhlmann, Meinard. 2012. "Quantum Field Theory." Stanford Encyclopedia of Philosophy. Last modified September 27, 2012.

https://plato.stanford.edu/entries/quantum-field-theory.

Lamoreux, Steve. 1997. "Demonstration of the Casimir Force in the 0.6 to $6 \mu \mathrm{m}$ Range." Physical Review Letters. 78:1. 5-8. https://doi.org/10.1103/PhysRevLett.78.5.

Leftow, Brian. 1991. Time and Eternity. New York, Ithica: Cornell University Press.

Leininger, Lisa. 2020. “Temporal B-Coming: Passage Without Presentness." Australasian Journal of Philosophy.

https://doi.org/10.1080/00048402.2020.1744673.

Maudlin, Tim. 1994. Quantum Non-Locality and Relativity: Metaphysical Intimations of Modern Physics. Massachusetts: Blackwell Publishers.

McCall, Storrs. 1994. A Model of the Universe: Space-Time, Probability, and Decision. Oxford: Clarendon. Oxford Scholarship Online.

Mullins, Ryan T. 2016. The End of the Timeless God. Oxford: Clarendon. Oxford Scholarship Online.

https://doi.org/10.1093/acprof:oso/9780198755180.001.0001.

Nikolic, Hjorve. 2005. "Bohmian Particle Trajectories in Relativistic Fermionic Quantum Field Theory." Foundations of Physics Letters. 18:2. 363-380. https://doi.org/10.1007/s10702-005-3957-3.

Padgett, Alan. 1992. God, Eternity and the Nature of Time. New York: St. Martin's Press. https://doi.org/10.1057/9780230376519.

Pylkkänen, Paavo. 2007. Mind, Matter and the Implicate Order. New York: Springer. Rogers, Catherine. 2007. Anselmian Eternalism: The Presence of a Timeless God. Faith and Philosophy. 24:1. 3-27. https://doi.org/10.5840/faithphil200724134.

Stapp, Henry. 1985. "Einstein Time and Process Time." In Physics and the Ultimate Significance of Time, edited by David R. Griffin. Princeton, New Jersey: University Press.

Zimmerman, Dean. 2001. "God Inside Time and Before Creation." In God and Time: Essays on the Divine Nature, edited by Gregory Ganssle, David Woodruff. Oxford University Press.

https://doi.org/10.1093/acprof:oso/9780195129656.003.0005.

Published Online: April 21, 2021 